\title{
Urgences
}

\section{Envers et contre tout}

\section{Jacquelin Marcheterre}

Numéro 23, avril 1989

Lisière du livre

URI : https://id.erudit.org/iderudit/025512ar

DOI : https://doi.org/10.7202/025512ar

Aller au sommaire du numéro

Éditeur(s)

Urgences

ISSN

0226-9554 (imprimé)

1927-3924 (numérique)

Découvrir la revue

Citer cet article

Marcheterre, J. (1989). Envers et contre tout. Urgences, (23), 22-25.

https://doi.org/10.7202/025512ar

Ce document est protégé par la loi sur le droit d'auteur. L'utilisation des services d'Érudit (y compris la reproduction) est assujettie à sa politique d'utilisation que vous pouvez consulter en ligne.

https://apropos.erudit.org/fr/usagers/politique-dutilisation/
Cet article est diffusé et préservé par Érudit.

Érudit est un consortium interuniversitaire sans but lucratif composé de l'Université de Montréal, l'Université Laval et l'Université du Québec à Montréal. Il a pour mission la promotion et la valorisation de la recherche. https://www.erudit.org/fr/ 


\section{JACQUELIN MARCHETERRE Envers et contre tout}

Que dois-je donc taire?

Que dois-je donc transposer?

Roger des Roches, Tout est normal, tout est terminé

L'unité contrastée de la maquette', la composition illustrée de la première de couverture excitent d'abord la curiosité, puis davantage le plaisir de l'amateur, le désir autrement dit, le privilège de la riposte critique: face au schématisme des couleurs, à la ciselure des arrangements graphiques, à la présence corrélative de figures, d'éléments paratextuels organisés, voire structurés.

La lettre blanche du titre, judicieusement placée, ici, à l'intérieur d'un rectangle qui est presque un carré, de couleur rouge et noire sur fond jaune, prédomine sur la lettre également blanche du nom de l'auteur; plus volumineuse, d'une lisibilité accrue par le procédé, isolée sur quatre lignes, étagée, la lettre se trouve prise, ni plus ni moins casée, répartie sur une surface qui s'apparente étrangement à la figure du damier ${ }^{2}$.

Lire le titre, le relire, prendre soudainement un parcours inattendu, fuir en avant, laisser ici la lecture en plan, la reprendre là au profit de telle case, de tel mot, c'est accorder déjà à la composition du titre, toute la force de recul du lacunaire. L'urgence de mener la lecture à une dame, l'initiative discrète, en effet prescrite par la liberté, l'invention et la règle du jeu forcent en un sens le déplacement, le passage d'une case à l'autre, l'avancée logique, restreinte; de là, la traversée restrictive du territoire titulaire: par omission, reprise du même, par rebond, différence... Bref, le damier, la forme de la figure, la totalité virtuellement accessible de son carrelage inclinent donc, en vertu d'une autorité paratextuelle, à épuiser, sinon à négocier avec tact la charge anagrammatique du titre $^{3}$.

Mais on y reviendra plus loin.

Au bas de la couverture, un cercle d'un format moindre fait aussi figure; il apparaît décentré par rapport au titre, logé sur la droite, juste au-dessous du damier. 
Le cercle dont on parle, traversé ici d'une barre oblique, symbolise de toute évidence l'interdiction. Que dire? De l'ordre du cliché, la contribution symbolique échappe de justesse à l'emprise toutefois redoutable de la banalité, puisqu'elle avoue de fait un petit défaut de fabrique: on remarque que le thème de l'interdit apparaît par-dessus la barre oblique... C'est là un vice amusant, mais à la fois une relance toute désignée, un détail somme toute, à la faveur néanmoins sérieuse d'un écart sous la commande duquel, on le constate, le symbole (nommons-le ainsi) opère mine de rien une subtile censure.

Alors? Où donc mènent le titre, l'essor promis par le dispositif titulaire, d'où peut provenir l'intérêt du symbole, de la trouvaille figurative? Que réserve la force cohésive d'un assemblage tel?

Par delà le principe, le lieu commun de l'interdiction, le symbole déplace l'interdit, il le retourne sur lui-même, il le brouille et, du coup, il souligne l'absence, la présence métaphorique de la femme, il met en relief le thème, l'objet censément visé par la censure. On finit par le voir. Le baiser qu'il arbore (l'empreinte, la trace grasse, craquelée, laissée par la bouche d'une femme imaginaire...) ne représente pas moins ce que la ligne horizontale, le gage de sensualité évacue à loisir: les lèvres (désignation charnière), le sexe convoité, inaccessible ou perdu, de la femme ${ }^{4}$. Le symbole accentue l'absence, reflétant tel un miroir le passage éphémère de la femme, la perte ou l'esquive... En réalité, le symbole illustre la charge subversive du détournement, il montre la disparition de la femme (clin d'oeil à la quatrième de couverture...); il place à découvert le geste critique dont il use, le rapt du regard d'où il puise, à partir duquel il assure l'habituelle efficacité qu'on lui reconnaît.

Le symbole montre plus qu'il ne cache en définitive; certes il opère une censure mais au sens strict du terme, produisant plutôt un effet de censure.

D'ailleurs il suffit de revenir au titre pour observer un effet du même genre. Coupé, morcelé par la forme du damier, le titre figure le repli, il prête autant de plis à la censure. Le titre résiste à la lecture, il dissimule, il recouvre un mot, un autre mot, une mine lexicale. À la longue, il autorise cependant une reformulation, au grand privilège d'un enjeu combinatoire qu'il ne cesse de trahir sous le coup d'une disposition graphique extrêmement fonctionnelle. Il suffit de relire la trame du damier pour s'en assurer. Sans toutefois forcer l'enjeu en question, la traversée de la zone titulaire 
favorise rapidement l'adhérence, la découverte d'un titre tout à fait réductible, d'un nouvel indicatif.

\section{Tout est mal, tout est miné}

issu de cases dont la correspondance, l'harmonie de la disposition ne font aucun doute. Le titre se délie, telle une langue, il parle en dépit des apparences, il articule autre chose, il révèle, sous l'effet d'une tension symétrique, une parole percutante dont la vérité assertive cautionne irréfutablement la composition symbolique de la page de couverture.

Le titre exprime une forme de réalisme déceptif (on y entend assez clairement: ça y est, c'est fini!); il établit bel et bien une relation d'équivalence (presque logique) entre la normalité (le naturel attendu de la Rupture amoureuse ${ }^{5}$ ) et l'achèvement (la fin, navrante on présume, d'une telle relation). C'est là toutefois une relation à travers laquelle le texte célibataire travaille, où il creuse son lit en sourdine... À vrai dire, tout se passe en creux. En vertu d'un principe basé sur la symétrie, l'accord graphique et syntaxique, le titre avoue néanmoins une adéquation plus discrète, une relation entre le mal (le dommage résultant d'habitude de la Rupture) et la charge affective (la douleur sentimentale, le déchirement intérieur, souterrain, qui l'accompagnent): il se fait l'expression, détournée peut-être, d'un vague regret, d'une sorte de remords refoulé face à la perte ou l'esquive de la femme... Mais le titre, par delà l'allusion romantique, ne désigne pas moins la composition symbolique comme le lieu sans conteste d'une perversion (à bien y regarder, tout est mal, à preuve l'interdiction, le retournement du thème de l'interdit...), et, du coup, il attire l'attention sur les dégâts formellement causés, il incline à prendre la formule littéralement au sérieux car la composition ruine le code, elle ne cesse d'altérer la norme, la fonction représentative du symbole (la frange paratextuelle de la page de couverture) au grand privilège de la femme, de ses lèvres, de son sexe (qu'on le veuille ou non, la Rupture n'est pas définitive, ici, tout en elle est miné... $)^{6}$.

\section{Envers et contre tout.}

1. J'analyse très brièvement la page de couverture du dernier livre de Roger Des Roches, Tout est normal, tout est terminé, revue Les herbes rouges, 1987, $30 \mathrm{p}$. La maquette et l'illustration (fait remarquable, signifiant en regard de la présente analyse) sont ici le résultat d'une composition tout à fait unique et personnelle de l'écrivain, poète et informaticien. À cet effet d'ailleurs, la preuve écrite apparaît, si je puis dire, au bas de la troisième de couverture. 
2. Ou, autre piste possible ici volontairement inexplorée, sur celle d'une espèce de scrabble mais mécanisé comme, par exemple, le sont les petites plaques métalliques où sont inscrits les chiffres des heures et des minutes sur le cadran à affichage numérique de tel radioréveil, plaques qui tournent et «tombent» pour donner l'heure. L'heure juste est alors celle où «tout est normal, tout est terminé», la virgule qui suit «normal» s'inversant en l'accent aigu qui termine «terminé» pour mieux en clore la scansion. Voir note 4. (Note d'A.G.)

3. Si je choisis le terme de «charge " ce n'est pas, loin de là, par pur hasard. Le titre est en effet chargé, tel un support, voire surchargé sur le plan anagrammatique; il est à son comble, il menace de se diviser, de se répartir, de se lire autrement. Déjà, il conduit à la verticalité, il reconstitue, en acrostiche donc, l'acronyme:

Tout est

Normal

Tout est terminé

Il heurte, il choque la linéarité, il désigne en même temps la charge, la tension explosive, la menace ou le danger sous le couvert duquel la lecture inévitablement se déroule et se réalise.

4. Ces traces de lèvres, ici noires (comme le fond des lettres qui forment les mots «tout est»), sont évidemment un rappel des seize traces de lèvres, là rouges diversement (et sous la lettre du titre de la revue, du nom de l'auteur et du titre du recueil) sur les première et quatrième de couverture des proses bien nommées Les lèvres de n'importe qui, revue Les herbes rouges, no 70 , 1978. Ce petit livre est d'ailleurs le seul de l'auteur à être muni d'une bande sur laquelle est portée l'inscription suivante: “CE TEXTE N'EST PAS UN TEXTE JUSTE». (Note d'A.G.)

5. Je ne fais que respecter ici la graphie du mot, du livre dans lequel on peut le repérer, le lire à quelques reprises. Je le prélève, je le reprends, en un sens, au bénéfice de l'analyse, à l'avantage surtout de la trame titulaire.

6. On peut d'ailleurs y lire quelque part (p. 19):

C'est que je n'avouerai que par écrit

Mon goût pour la Rupture

C'est là une manière d'aveu, à la faveur, bien entendu, de l'écriture, de la Rupture; un aveu détourné cependant, puisqu'il conforte allusivement un autre intérêt, non moins percutant, pour la dissimulation; un tel aveu confirme, en filigrane, le pouvoir opérant de la Rupture, ce qu'elle autorise à taire, vu son ampleur, ce qu'elle permet de transposer, de dire tout autrement: un tel aveu rehausse la vertu masquante du paratexte donc. 